\title{
The Hakoniwa method, an approach to predict material properties based on statistical thermodynamics and ab initio calculations
}

Eiji Kamiyama ${ }^{1,2}$, a), Ryo Matsutani ${ }^{1}$, Ryo Suwa ${ }^{1}$, Jan Vanhellemont ${ }^{3}$ and Koji Sueoka ${ }^{1}$

${ }^{1}$ Department of Communication Engineering, Okayama Prefectural University, 111 Kuboki, Soja-shi, Okayama 719-1197, Japan

${ }^{2}$ Technology, GlobalWafers Japan Co., Ltd., 6-861-5 Higashiko, Seiro, Niigata 957-0197, Japan

${ }^{3}$ Department of Solid State Sciences, Ghent University, Krijgslaan 281-S1, Ghent B-9000, Belgium

\section{Abstract}

An approach based on statistical thermodynamics and ab initio calculations to predict properties of materials composed of different types of atoms is presented. The key point of what the authors called the "Hakoniwa" method, is to take into account all possible structural supercells constructed by the fixed number of atoms of each species according to the composition of the target material. The conservation of the total number of atoms enables calculating the average value of a material property for a given temperature by applying statistical thermodynamics to the material property values obtained for each of

a) E-mail: ejkamiyama@aol.com, Phone: +81-866-94-2136 
the possible supercells. The application of the Hakoniwa method is illustrated by calculating the average energy gain by mixing Sn and Si atoms in a Ge matrix, as function of the $\mathrm{Ge}_{1-\mathrm{x}-\mathrm{y}} \mathrm{Sn}_{\mathrm{x}} \mathrm{Si}_{\mathrm{y}}$ composition. The relative stability of each composition is compared allowing predicting the impact of Si doping on the stability of $\mathrm{Ge}_{1-\mathrm{x}-\mathrm{y}} \mathrm{Sn}_{\mathrm{x}} \mathrm{Si}_{\mathrm{y}}$ films epitaxially grown on a Ge substrate. In addition, the average bandgap is calculated for a given $\mathrm{Ge}_{1-\mathrm{x}-\mathrm{y}} \mathrm{Sn}_{\mathrm{x}} \mathrm{Si}_{\mathrm{y}}$ composition as a function of the temperature.

\section{Keywords}

statistical thermodynamics, ab initio calculation, average, supercell, Ge1-x-y $\mathrm{Sn}_{\mathrm{x}} \mathrm{Si}_{\mathrm{y}}$, bandgap 


\section{Introduction}

The stability of semiconductor alloys during hetero-epitaxial film growth at a given temperature is critical to be able to use bandgap engineering to obtain specific devices characteristics. The present paper discusses and demonstrates a statistical thermodynamics and ab initio calculation based method to predict composite material properties using all possible supercells with the given material composition, that the authors named the "Hakoniwa" method. The application of the Hakoniwa method is illustrated for the evaluation of the effect of Si doping on the stability of $\mathrm{Ge}_{1-\mathrm{x}} \mathrm{Sn}_{\mathrm{x}}$ films and on their bandgap. This type of films is studied intensively for their use in strained $\mathrm{Ge}_{1-\mathrm{x}} \mathrm{Sn}_{\mathrm{x}}$ quantum well channels on Ge substrates to take advantage of both carrier mobility enhancement [1] and bandgap reduction [2]. The Hakoniwa method allows calculating in a straightforward way the average value of material parameters that can be obtained by ab initio calculation. The method is based on the assumption that each value of a material parameter can be linked directly to a particular supercell using ab initio calculation which is not the case for the cluster expansion (CE) method [3].

Ab initio calculation can not only directly calculate bulk properties but also allows estimating various properties of impurities both in elemental crystals and in alloys by using the supercell method [4]. Applying the supercell method to 
semiconductor alloys, however, leads to a drastic increase of the number of possible configurations compared to the case where a single point defect in a supercell consisting of only one type of atoms is investigated. Some attempts have been made to estimate the properties of semiconductor alloys by calculating a specific model like a quasi-random configuration cell [5-8] for a given composition of atoms although not all possible configurations were examined. The quasi-random configuration approach is correct if the temperature of crystal growth is so high that the atoms can arrange themselves at random during growth. However, this is not possible when the crystal growth temperature is rather low as is the case for some epitaxial film growth processes like e.g. $\mathrm{Ge}_{1-\mathrm{x}} \mathrm{Sn}_{\mathrm{x}}$ on $\mathrm{Ge}$ [9]. The method that is described in the present paper considers all possible configurations for the supercell size that is chosen and, based on that, calculates the average material parameter for a given temperature. Using the proposed Hakoniwa method, the most likely configurations can be obtained without any parameter fitting or additional assumptions.

The application of the Hakoniwa method is illustrated for $\mathrm{Ge}_{1-\mathrm{x}-\mathrm{y}} \mathrm{Sn}_{\mathrm{x}} \mathrm{Si}_{\mathrm{y}}$ epitaxial growth on Ge and is used to estimate the average formation energy and the bandgap based on all possible configurations in a supercell containing a given number of Ge, Sn and Si atoms. This allows comparing the relative stabilities and bandgaps at various 
substrate temperatures which is useful for designing a $\mathrm{Ge}_{1-\mathrm{x}-\mathrm{y}} \mathrm{Sn}_{\mathrm{x}} \mathrm{Si}_{\mathrm{y}}$ epitaxial film growth process. Furthermore, the Hakoniwa method is used to investigate also the effect of the level of Si doping and the growth temperature.

\section{Principle of the Hakoniwa Method}

\section{2-1. Overview}

"Hakoniwa" is a Japanese traditional play creating "miniature gardens", by arranging a collection of beautiful objects in different ways in order to express something. The procedure proposed in the present paper is somewhat similar whereby the miniature garden is the supercell in which the different atoms, the "beautiful objects” are arranged in order to estimate a given-material parameter. A set of supercells of all possible atom configurations for a given supercell size with the target composition is first prepared. In order to estimate the properties of the material at a given temperature, the probability of each of the possible configurations is calculated in a statistical way. By using this approach, one can also obtain an insight in the dominant configuration of atoms for the macroscopic phenomenon in the real material that is being investigated. 
The main advantages of the "Hakoniwa" method are the following:

[i] Representation of the configuration of atoms is straightforward since they are located in a supercell as is commonly used for ab initio calculation. For each temperature, the dominant configurations are then determined by applying conventional statistical thermodynamics using the calculated formation energy and the weight of each irreducible configuration ( $=$ the total number of equivalent configurations). The formation energy and the weight obtained for each configuration allow determining the configurations which are dominant for the given composition and temperature.

[ii] Pseudomorphic hetero-epitaxial film growth can be considered by treating an anisotropically shaped supercell as will be illustrated further for epitaxial $\mathrm{Ge}_{1-\mathrm{x}-\mathrm{y}} \mathrm{Sn}_{\mathrm{x}} \mathrm{Si}_{\mathrm{y}}$ films on a Ge substrate.

[iii] In case that experimental values of the calculated material parameter are available, the comparison between experiment and calculation based on a chosen set of supercells will increase the confidence of the assumed structural origin of a physical phenomenon at a specific temperature. If there is no good agreement between the experimental values and the calculated ones, it is probable that the chosen supercell (size, composition, perhaps also some of the atoms not on substitutional positions, ...) was not suitable. The whole Hakoniwa procedure might then be repeated assuming a different supercell to 
start with.

The compositions and structures that are used are somewhat similar to atoms that are arranged in several ways inside a supercell in order to replicate a "macroscopic" crystal. The supercell used in the usual method can represent objects of all kinds in a crystal but is not always corresponding well to a macroscopic phenomenon that can be observed in actual material, and the obtained calculated result always depends on how the supercell was defined. There is therefore an uncertainty gap in the understanding and predicting of properties of actual materials using models based on the use of supercells with a limited number of atoms. The Hakoniwa method enables to overcome this problem to a large extent by evaluating the probability of each configuration of the objects in the supercells as a replica of the micro structure of the real material.

\section{2-2. The Hakoniwa method procedure}

The detailed procedure of the Hakoniwa method is as follows:

[A] Determine the size of the supercells that will be used to approximate the real crystal by superposition of a limited set of them, similar with the number of atoms in the maximum size cluster to be selected in the CE method.

[B] Choose the number of atoms of each species in the set of supercells according to the 
composition of the target material. The conservation of total number of atoms allows calculating the average material parameter values statistically in step I, using a microcanonical ensemble.

[C] Determine all possible configurations of the atoms in the supercell.

[D] Extract the set of base cells (= corresponding with the irreducible configurations of Table I) from step C based on the symmetry and periodic conditions of the cell and calculate the weight of each irreducible configuration.

[E] Optimize the geometry of all the base cells and obtain the total energy for each base cell.

[F] Calculate the formation energy $E_{f}^{i}$ of the $\mathrm{i}^{\text {th }}$ supercell by the standard procedure using ab initio calculation, see e.g. [4] using the total energies and the chemical potential of atoms in the supercells.

[G] Calculate the probability $p^{i}$ of each base cell from the formation energies of all the base cells taking their weight $w^{i}$ into consideration.

[H] Calculate the target material parameter $M^{i}$ for all the base cells.

[I] Calculate the average of the material parameter $\bar{M}$ by using the probabilities obtained in step G.

Similar to the expansion degrees in CE method, the size of the base supercell 
determines the accuracy of the calculated results and of the range of atom concentrations that can be studied in a composite material whereby the calculation costs increase very rapidly as the supercell size increases. Besides calculation of alloy properties, the Hakoniwa method can in principle also be used to calculate average properties related to impurity complexes or clusters. It is also possible to include a low concentration of intrinsic point defects by using a larger cell to examine their impact and behavior, although even in that case, the calculated properties may still show a base supercell size dependence similar to that obtained with the usual supercell method. Steps A to G are similar to the SOD (Site-Occupancy Disorder) method [10] while steps $\mathrm{H}$ and I are an application of statistical thermodynamics on the results obtained in steps A to G. Using statistical thermodynamics, the probability $p^{i}$ can be written as:

$$
p^{i}=\frac{1}{Z} w^{i} \exp \left[-\frac{E_{f}^{i}}{k_{B} T}\right]
$$

with $w^{i}$ the statistical weight of the $\mathrm{i}^{\text {th }}$ supercell, $k_{B}$ the Boltzmann constant and $T$ the absolute temperature. $Z$ is the microcanonical ensemble partition function given by:

$$
Z=\sum_{i} w^{i} \exp \left[-\frac{E_{f}^{i}}{k_{B} T}\right]
$$

If the targeted material parameter $M^{i}$ for the i-th supercell can be calculated, the average value of that material parameter is given by:

$$
\bar{M}=\sum_{i} p^{i} \cdot M^{i}
$$


$E_{f}{ }^{i}$ in equations (1) and (2) also can be replaced by the total energies, which was done by Grau-Cresoi et al. [8], for the calculated cells in the step E since changes of the energy reference for $E_{f}^{i}$ have no effect in (eq. 1). It will be useful, however, to adjust the energy reference to the chemical potential of atoms and use standard $E_{f}^{i}$ to evaluate the relative probability of each possible cell structure for different compositions as will be discussed in section 3.4.

One of the most important advantages of the Hakoniwa method is that it enables to obtain average values of a physical parameter at a given temperature in a statistical way by using equations (1)-(3) if one can obtain the material parameter value for each of the possible irreducible configurations of the supercell with a given composition. Similar equations have also been applied to finite sites of contamination atoms in a macroscopic system (without a periodic condition), e.g. to describe the metal gettering phenomenon in a silicon wafer [11].

The formation energy $E_{f}^{i}$, defined in the frame of the supercell method as mentioned in step F, also appears in the exponential term of grand canonical ensemble partition function $\Xi$ that can be written in a general form as

$$
\Xi=\sum_{j} \sum_{i} \exp \left[-\frac{\left(E_{i j}-\mu N_{i}\right)}{k_{B} T}\right]
$$

where $E_{i j}$, is the energy at the state $j$ (each atomic configuration) of the number of the 
particles $N_{i}$, and $\mu$ is the chemical potential. The Hakoniwa method in principle allows predicting the most stable average composition and structure of a material by using eq. 4 within the finite size of the supercells and their superposition (averaging weighed by their probability $p_{i}$ ). This is, however, beyond the scope of the present paper which is mainly aiming to understand hetero-epitaxial film growth with a fixed ratio of atom species.

\section{Example of application: epitaxial $\mathrm{Ge}_{1-\mathrm{x}-\mathrm{y}} \mathrm{Sn}_{\mathrm{x}} \mathrm{Si}_{\mathrm{y}}$ film growth on a Ge substrate}

\section{3-1. Model construction (from step A to D)}

First, as an illustration, the 2x2x2 supercell of the Ge primitive cell (16 atoms) is selected as the supercell size (Step A). The number of atoms of each species in step B is given by the total number of atoms in the cell, and for present example is sixteen. For illustration purposes only, the number of Sn atoms per cell is fixed to 1, corresponding with a Sn concentration of about 7\%, and the number of Si atoms is varied between 1 and 6. In steps $\mathrm{C}$ and $\mathrm{D}$, all irreducible configurations of the number of $\mathrm{Sn} / \mathrm{Si}$ atoms in a 16-Ge atoms supercell are determined with their respective weights (= the number of equivalent supercells with the same irreducible configuration). The results are 
summarized in Table I, for the given symmetry of the crystal and for the periodicity of the cell. This search can be automatically performed by using a script program code based on a published algorithm [12], thus reducing drastically the calculation cost.

Before step C, the possible positions for each atom must be defined similar as is done in the conventional supercell method. Substitutional sites for a Si or a Sn atom in a Ge lattice have a formation energy $\left(E_{f}\right)$ around $0.3 \mathrm{eV}$ which is much lower than that for interstitial sites which is more than $3 \mathrm{eV}$ as illustrated in Table II for 64 atom supercells. $\mathrm{Ab}$ initio calculations are performed for Si and Sn atoms in a substitutional and in a tetrahedral interstitial site using the standard procedure. The increase of the formation energy for the 16-atom cell is due to the higher concentration effect. Only the substitutional positions should therefore be taken into account for constructing all the possible structures to describe the $\mathrm{Ge}_{1-\mathrm{x}-\mathrm{y}} \mathrm{Sn}_{\mathrm{x}} \mathrm{Si}_{\mathrm{y}}$ film growth as those with interstitial atoms have a much higher energy as shown in Table II and are therefore very unlikely to occur. In general, if a stable interstitial site would become a candidate for describing a system due to a lower formation energy, which is not the case for the $\mathrm{Ge}_{1-\mathrm{x}-\mathrm{y}} \mathrm{Sn}_{\mathrm{x}} \mathrm{Si}_{\mathrm{y}}$ system in the present study, such a position should also be included. It should be noted that interstitial atoms play an important role in many defect related phenomena in semiconductor materials like $\mathrm{Si}$, Ge and their alloys, which all have the low atom 
density diamond structure.

\section{3-2. Ab initio calculation details (Step E)}

For geometry optimization of the base cells in step E, the cell size is fixed as that of the un-doped Ge cell with P1 symmetry as the goal is to investigate $\mathrm{Ge}_{1-\mathrm{x}-\mathrm{y}} \mathrm{Sn}_{\mathrm{x}} \mathrm{Si}_{\mathrm{y}}$ hetero-epitaxial growth on a Ge substrate. When one wants to calculate the lattice constant of the $\mathrm{Ge}_{\mathrm{x}} \mathrm{Sn}_{\mathrm{y}} \mathrm{Si}_{\mathrm{z}}$ alloy, however, the cell size should also be allowed to relax. To investigate pseudomorphic epitaxial growth, only the z-axis perpendicular to the substrate is relaxed.

All conditions of the ab initio calculation in step $\mathrm{E}$ and $\mathrm{H}$ of the Hakoniwa method can be set as in the conventional approach used for ab initio calculation using the supercell method. The ab initio calculations in the present study are also based on that standard approach, using the local density approximation $[13,14]$ with the ultrasoft pseudopotential method [15], and plane waves as basis set for efficient structure optimization. The expression proposed by Perdew et. al. [16] is used for the exchangecorrelation energy in the generalized gradient approximation (GGA) in the atom relaxation. The screened exchange local density approximation (sX-LDA) method as proposed by Seidl [17] and by Bylander and Klienman [18] is used for the bandgap 
calculations as an example of the step G, as will be discussed further in section 3-5. This sX-LDA method provides band structures that are in very good agreement with experimental results [19]. The CASTEP code is used to self-consistently solve the Kohn-Sham equation using a three-dimensional periodic boundary condition [20]. The density mixing method [21] and the BFGS geometry optimization method [22] are used to optimize the electronic structure and the atomic configurations, respectively. For kpoint sampling, the $4 \times 4 \times 4$ special points of the Monkhorst-Pack grid are used [23]. The cutoff energy of the plane waves is $340 \mathrm{eV}$ for the atom relaxations and $440 \mathrm{eV}$ for the band calculation. In the band calculation, the symmetry of the atomic configuration in each irreducible configuration is also being considered to estimate the bandgap in section 3-5.

\section{3-3. Formation energy, probability and weights (step F and G)}

After the geometry optimization, one can calculate $E_{f}$ of all the base cells (Step F). Figure 1 illustrates this and shows the different $E_{f}$ values with the corresponding weights and probabilities (Step H) for a Si atom content $\mathrm{x}=2$ in the 16 atom supercell corresponding with a $\mathrm{Ge}_{13 / 16} \mathrm{Sn}_{1 / 16} \mathrm{Si}_{2 / 16}$ material. Some structures have higher probabilities at a lower temperature and will therefore tend to be formed during low 
temperature mixed alloy epitaxial layer growth. When the material parameter that is being investigated has been calculated for all the base cells, its average value can be calculated using eq. 3, taking into account the relative weights of all base cells.

\section{3-4. Results on energy gain (step $H$ and I) and implications for epitaxial film}

growth

An example of a material parameter that can be calculated is $E_{f}$ itself which

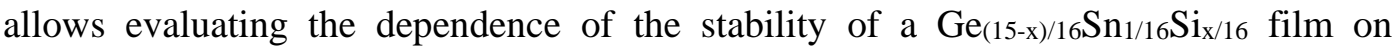
temperature and composition. Using the average $E_{f}$, the energy gain dependence on the Si atom content $\mathrm{x}$ in the 16 atom supercell is calculated between 200 and $600 \mathrm{~K}$ and the results are shown in Fig. 2 by comparing the average $E_{f}$ to the $E_{f}$ of an isolated $\mathrm{Si} / \mathrm{Sn}$ atom (shown in Table II) as reference energy. The positive correlation of the energy

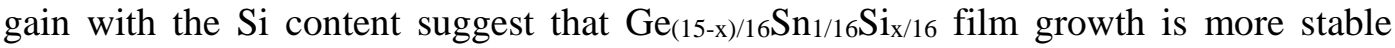
when the intensity of the Si atom beam in the reactor is increased and/or the temperature is decreased during molecular beam epitaxy (MBE). The energy gain increases generally when the temperature decreases. The structures in supercells having lower $E_{f}$ at lower temperature will therefore be dominant in hetero-epitaxial films grown at relatively low temperature. Since the present calculation did not take the dynamics into 
account and ignored also the barriers for migration of atoms, the results shown in Fig. 1 do not directly correspond to the process window in which high quality epitaxial single crystal film growth is possible in an actual MBE system. Dynamical aspects can however be studied using a similar scheme as was discussed elsewhere [24]. The slopes of all lines in Fig. 2 decrease when $\mathrm{x}$ is larger than 4. This agrees well with other theoretical and experimental reports on experimental $\mathrm{Ge}(15-\mathrm{x}) / 16 \mathrm{Sn}_{1 / 16} \mathrm{Si}_{\mathrm{x} / 16}$ heteroepitaxial growth, in which a Si/Sn atomic ratio of 4 was found to be suitable for pseudomorphic epitaxial growth on Ge substrate [25].

When one considers reactions of $\mathrm{Sn}$ and $\mathrm{Si}$ atoms on the surface of the Ge substrate, one can obtain the relative "existence probability” of the 16 atom Ge based supercells containing $\mathrm{x}$ Si atoms and one Sn atom based on the calculated energy gain $\dot{E}^{x}$ as follows:

$$
\left[S i_{x} S n_{1}\right]=C_{x} \cdot[S i]^{x} \cdot[S n] \cdot \exp \left[\frac{\dot{E}^{x}}{k_{B} T}\right]
$$

where $C_{x}$ is a constant for each $x$ and $[S i]$ and $[S n]$ are atomic concentrations in the grown film with composition $\mathrm{Ge}_{(15-\mathrm{x}) / 16} \mathrm{Sn}_{1 / 16} \mathrm{Si}_{\mathrm{x} / 16}$. These concentrations are proportional to the amounts of $\mathrm{Si}$ and $\mathrm{Sn}$ atoms supplied onto the Ge substrate at temperature $T$ in a reaction chamber like that of a MBE system. Figure 3 shows the calculated exponential term in eq. 5, normalized with respect to the value at $600 \mathrm{~K}$. This 
figure predicts that the Sn content in the grown film increases due to clustering of Si and Sn atoms when the substrate temperature is relatively low. Note that the larger $\mathrm{x}$ is, the larger the existence probabilities are, due to the larger energy gains. A similar phenomenon will occur between a vacancy and a $\mathrm{Sn}$ atom during $\mathrm{Ge}_{1-\mathrm{x}} \mathrm{Sn}_{\mathrm{x}}$ film growth. The increase of incorporated vacancies in the Ge matrix was actually observed during epitaxial $\mathrm{Ge}_{1-\mathrm{x}} \mathrm{Sn}_{\mathrm{x}}$ film growth at lower temperatures [26].

\section{3-5. Result of bandgap (step $H$ and I)}

Finally, also as an illustration, the calculated average bandgap and its standard deviation as function of the growth temperature of a $\mathrm{Ge}_{13 / 16} \mathrm{Sn}_{1 / 16} \mathrm{Si}_{2 / 16}$ film, is shown in Fig. 4. The calculated band structures for all the base cells for this composition show direct bandgaps. The average bandgap decreases with increasing temperature.

\section{Summary}

The application of the proposed Hakoniwa method is demonstrated by evaluating the Si doping effect on the stability and on the bandgap of $\mathrm{Ge}_{(15-}$ x)/16 $\mathrm{Sn}_{1 / 16} \mathrm{Si}_{\mathrm{x} / 16}$ films using 16 atom Ge based supercells containing one Sn atom and $\mathrm{x} \mathrm{Si}$ 
atoms. When applying this method, one should take into account all possible structural supercells constructed by the number of atoms of each species according to the composition of the target material. The Hakoniwa method can provide the average value of a physical parameter at a finite temperature by calculating it using statistical thermodynamics based on the values of that physical parameter obtained for each of the possible supercells using ab initio calculation. 


\section{Acknowledgement}

This work is partially supported by the ALCA program from JST in Japan. The authors would like to thank Dr. Abhijit Chatterjee of Dassault Systemes Biovia K.K. for stimulating discussions and for developing the script program code that is used in the present study to calculate the irreducible atom configurations. 


\section{REFERENCES}

1. J. D. Sau and M. L. Cohen, Phys. Rev. B 75 (2007) 045208.

2. Y. Chibane and M. Ferhat, J. Appl. Phys. 107 (2010) 053512.

3. J. M. Sanchez, F. Ducastelle and D. Gratias, Physica A 128 (1984) 334.

4. R. M. Nieminen, Theory of Defects in Semiconductors, David A. Drabold and Stefan

K. Estreicher (Eds.), Springer-Verlag Berlin Heidelberg 2007, pp 29-68.

5. M. Ferhat and F. Bechstedt, Phys. Rev. B 65 (2002) 075213.

6. A. Chroneos, C. Jiang, R. W. Grimes and U. Schwingenschlögl, Chem. Phys. Lett. $493(2010) 97$.

7. A. Chroneos, C. Jiang, R. W. Grimes, U. Schwingenschlögl, and H. Bracht, Appl. Phys. Lett. 94 (2009) 252104.

8. A. Chroneos, C. Jiang, R. W. Grimes, U. Schwingenschlögl, and H. Bracht, Appl. Phys. Lett. 95 (2009) 112101.

9. Y. Shimura, N. Tsutsui, O. Nakatsuka, A. Sakai and S. Zaima, Thin Solid Films 518 (2010) S2.

10. R. Grau-Crespo, S. Hamad, C. R. A. Catlow and N. H. de Leeuw, J. Phys. Condens. Matter. 19 (2007) 256201.

11. E. Kamiyama, K. Sueoka and J. Vanhellemont, ECS Journal of Solid State Science 
and Technology 4 (2015) 232.

12. J. L. Gavartin, M. Sarwar, D. C. Papageorgopoulos, D. Gunn, S. Garcia, A. Perlov, A. Krzystala, D. L. Ormsby, D. Thompsett, G. Goldbeck-Wood, A. Andersen and S. French, ECS Trans. 25 (2009) 1335.

13. P. Hohenberg and W. Kohn, Phys. Rev. 136 (1964) B864.

14. W. Kohn and L. Sham, Phys. Rev. 140 (1965) A1133.

15. D. Vanderbilt, Phys. Rev. B 41 (1990) 7892.

16. J.P. Perdew, K. Burke and M. Ernzerhof, Phys. Rev. Lett. 77 (1996) 3865.

17. A. Seidl, A. Görling, P. Vogl, J. A. Majewski and M. Levy, Phys. Rev. B 53 (1996) 3764.

18. B. M. Bylander and L. Kleinman, Phys. Rev. B 41 (1990) 7868.

19. K. Mistry, C. Allen, C. Auth, B. Beattie, D. Bergstrom, M. Bost, M. Brazier, M. Buehler, A. Cappellani, R. Chau, C.-H. Choi, G. Ding, K. Fischer, T. Ghani, R. Grover, W. Han, D. Hanken, M. Hattendorf, J. He, J. Hicks, R. Huessner, D. Ingerly, P. Jain, R. James, L. Jong, S. Joshi, C. Kenyon, K. Kuhn, K. Lee, H. Liu, J. Maiz, B. McIntyre, P. Moon, J. Neirynck, S. Pae, C. Parker, D. Parsons, C. Prasad, L. Pipes, M. Prince, P. Ranade, T. Reynolds, J. Sandford, L. Shifren, J. Sebastian, J. Seiple, D. Simon, S. Sivakumar, P. Smith, C. Thomas, T. Troeger, P. Vandervoorn, S. Williams, and K. 
Zawadzki, Proc. IEDM (2007) 247.

20. S. J. Clark, M. D. Segall, C. J. Pikard, P. J. Hasnip, M. J. Robert, K. Refson and M.C. Payne, Z. Krist. 220 (2005) 567.

21. G. Kresse and J. Furthmuller, Phys. Rev. B54 (1996) 11169.

22. T. Fischer and J. Almlof, J. Phys. Chem. 96 (1992) 9768.

23. H. Monkhorst and J. Pack, Phys. Rev. B13 (1976) 5188.

24. E. Kamiyama, J. Vanhellemont and K. Sueoka, Phys. Status Solidi B 251 (2014) 2185.

25. Y.-Y. Fang, J. Xie, J. Tolle, R. Roucka, V. R. D’Costa, A. V. G. Chizmeshya, J. Menendez and J. Kouvetakis, J. Am. Chem. Soc. 130 (2008) 16095.

26. E. Kamiyama, S. Nakagawa, K. Sueoka, T. Ohmura, T. Asano, O. Nakatsuka, N. Taoka, S. Zaima, K. Izunome and K. Kashima, Appl. Phys. Express 7 (2014) 021302. 


\section{FIGURE CAPTIONS}

Fig. 1 (Color online) Formation energies $E_{f}$ of supercells with their weights and probabil

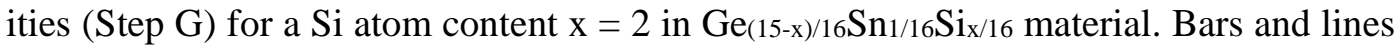
show formation energies (right vertical axis) and probabilities (left vertical axis) with th eir weights (the horizontal axis), respectively. Structures in supercells having lower $E_{f}$ at lower temperature are dominant in real hetero-epitaxial layers. The lines connecting the discrete probability points are only drawn to guide the eye.

Fig. 2 (Color online) Energy gain dependence on Si atom content x, calculated at differe nt temperatures compared to the formation energy $E_{f}$ of the isolated Si/Sn atom as a refe rence.

Fig. 3 (Color online) Calculated exponential term in eq. 5, normalized with respect to th e value at $600 \mathrm{~K}$.

Fig. 4 (Color online) Calculated dependence of band gap $E_{g}$ and its standard deviation on the substrate temperature during $\mathrm{Ge}_{(13 / 16)} \mathrm{Sn}_{(1 / 16)} \mathrm{Si}_{(2 / 16)}$ film growth. 
Table I. Number of atom configurations of a 16 atom Ge supercell containing 1 Sn atom and up to 6 Si atoms.

\begin{tabular}{|c|c|c|}
\hline Compositions & $\begin{array}{c}\text { Irreducible } \\
\text { Configurations }\end{array}$ & Total Configurations \\
\hline $\mathrm{Ge}_{15 / 16} \mathrm{Sn}_{1 / 16}$ & 1 & $16\left(={ }_{16} \mathrm{C}_{1}\right)$ \\
\hline $\mathrm{Ge}_{14 / 16} \mathrm{Sn}_{1 / 16} \mathrm{Si}_{1 / 16}$ & 4 & $240\left(={ }_{16} \mathrm{C}_{2}\right)$ \\
\hline $\mathrm{Ge}_{13 / 16} \mathrm{Sn}_{1 / 16} \mathrm{Si}_{2 / 16}$ & 13 & $1680\left(={ }_{16} \mathrm{C}_{3}\right)$ \\
\hline $\mathrm{Ge}_{12 / 16} \mathrm{Sn}_{1 / 16} \mathrm{Si}_{3 / 16}$ & 39 & $21840\left(={ }_{16} \mathrm{C}_{5}\right)$ \\
\hline $\mathrm{Ge}_{11 / 16} \mathrm{Sn}_{1 / 16} \mathrm{Si}_{4 / 16}$ & 97 & $48048\left(={ }_{16} \mathrm{C}_{6}\right)$ \\
\hline $\mathrm{Ge}_{10 / 16} \mathrm{Sn}_{1 / 16} \mathrm{Si}_{5 / 16}$ & 187 & $80080\left(={ }_{16} \mathrm{C}_{7}\right)$ \\
\hline $\mathrm{Ge}_{9 / 16} \mathrm{Sn}_{1 / 16} \mathrm{Si}_{6 / 16}$ & 290 &
\end{tabular}


Table II. Calculated formation energies $E_{f}$ of a $S i$ and a $S n$ atom at a substitutional (S) or a tetrahedral ( $\mathrm{T})$ site in a Ge crystal.

\begin{tabular}{|c|c|c|c|}
\hline \multirow{2}{*}{ Si site } & \multicolumn{3}{|c|}{ Formation energy $\left(E_{f}\right)(\mathrm{eV})$} \\
\cline { 2 - 4 } & 16 atom-cell & 64 atom- cell & 216 atom-cell \\
\hline Si (S) & 0.11 & 0.07 & 0.07 \\
\hline Si (T) & - & 3.11 & - \\
\hline Sn (S) & 0.33 & 0.22 & 0.21 \\
\hline Sn (T) & - & 3.60 & - \\
\hline
\end{tabular}

Table II Kamiyama et al. 


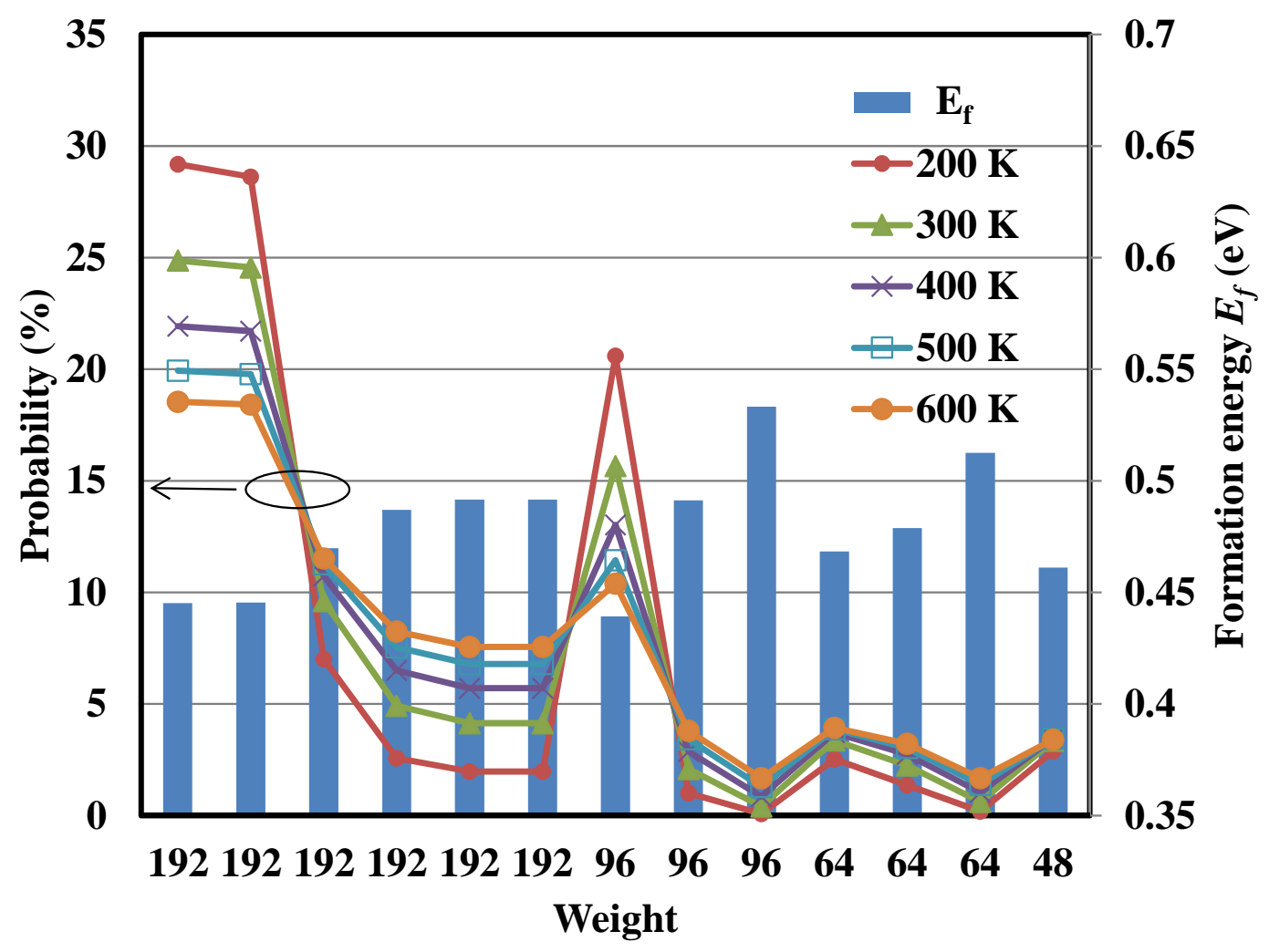

Fig 1 Kamiyama et al. 


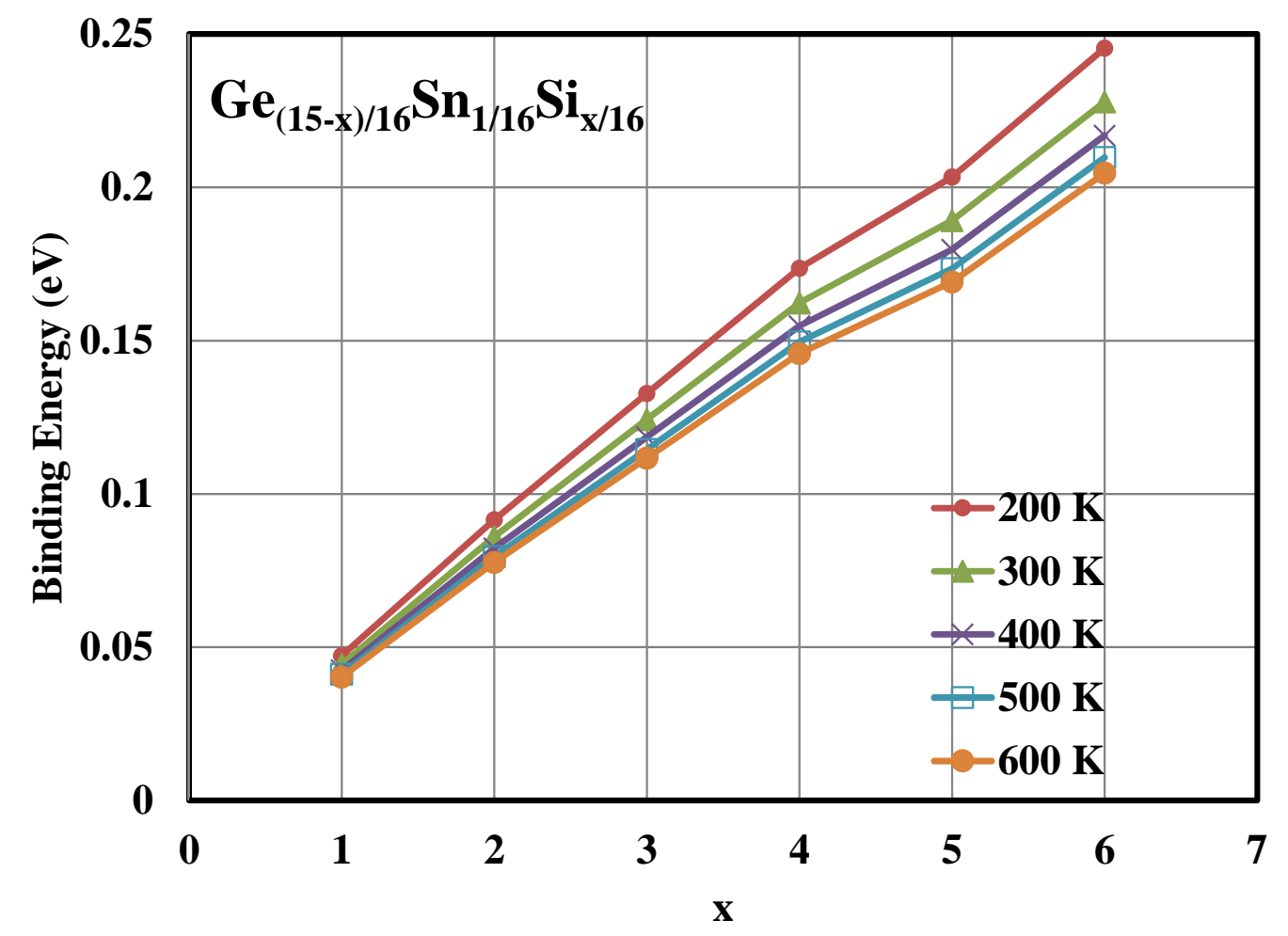

Fig 2 Kamiyama et al. 


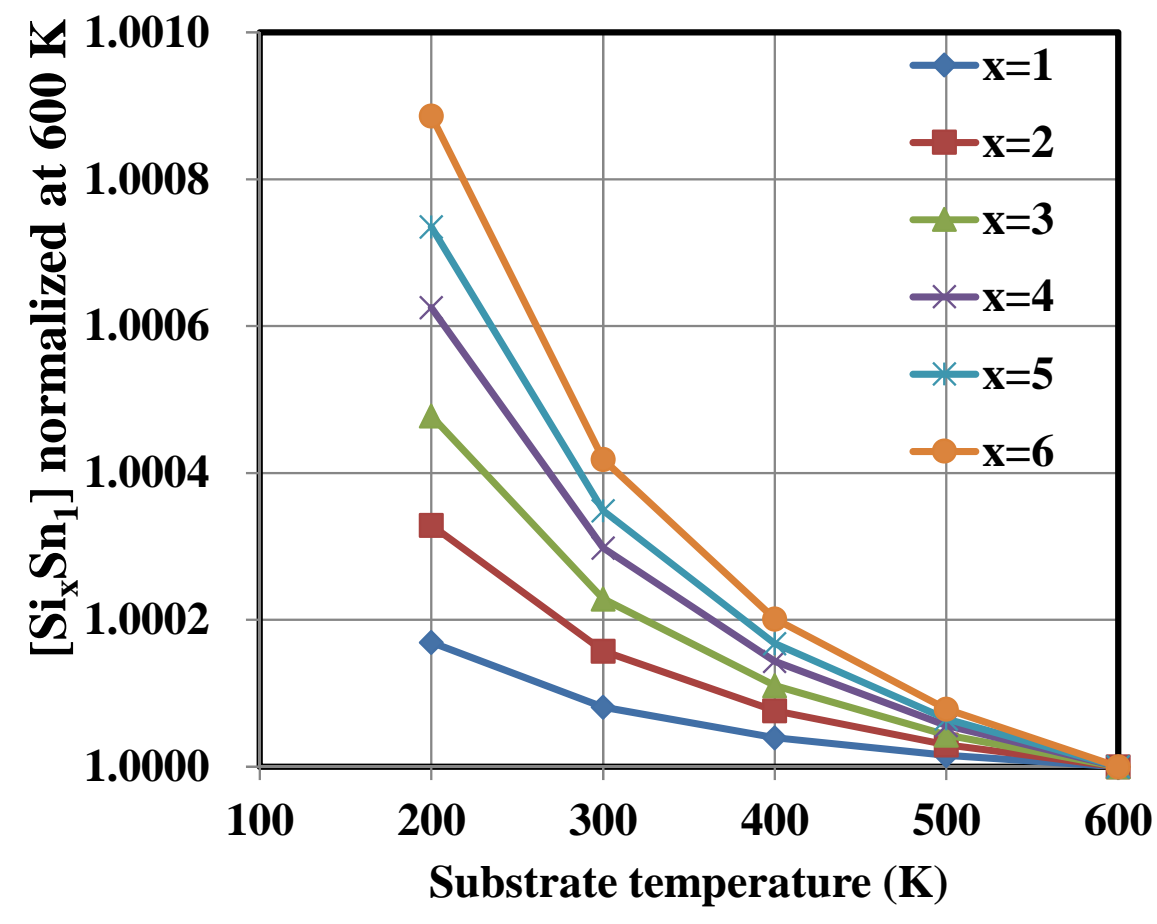

Fig 3 Kamiyama et al. 


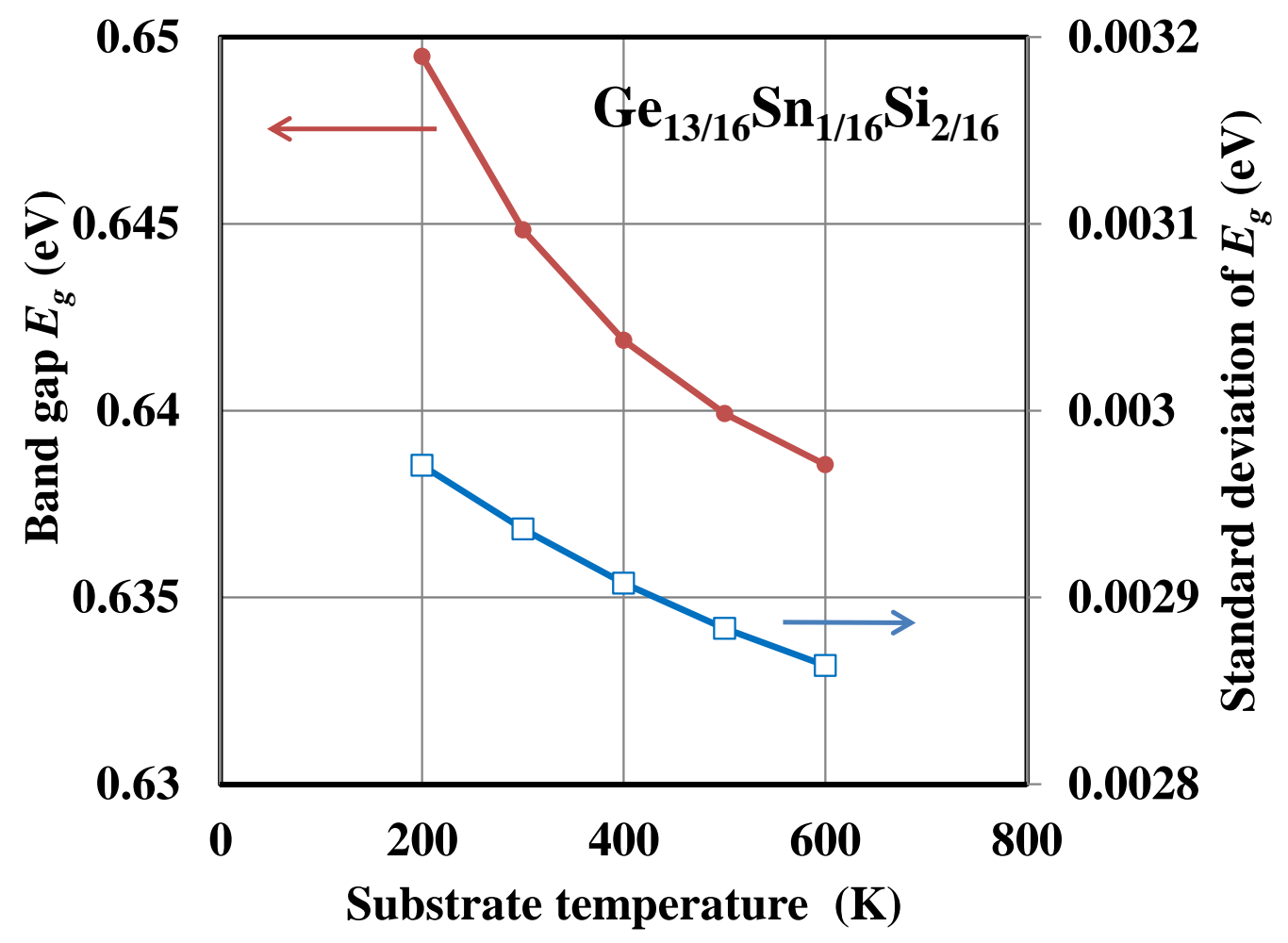

Fig 4 Kamiyama et al. 